\title{
Editor's Note: Special Issue on Big Data Analytics and Privacy
}

Published online: 6 September 2019

(C) Springer Science+Business Media, LLC, part of Springer Nature 2019

Journal of Signal Processing Systems gratefully acknowledges the editorial work of the scholar listed below on the special issue entitled "Big Data Analytics and Privacy".

Meikang Qiu

Columbia University

USA

Publisher's Note Springer Nature remains neutral with regard to jurisdictional claims in published maps and institutional affiliations. 